\title{
Pulseless Electrical Activity Arrest as the First Symptom of Testicular Cancer with Subsequent Phlegmasia Cerulea Dolens
}

\author{
Shane Belvedere ${ }^{1^{*}}$ \\ 1 Department of Vascular Surgery, Royal Melbourne Hospital, 300 Grattan Street, Parkville, Victoria, Australia
}

\begin{abstract}
Introduction: Phlegmasia cerulea dolens (PCD) is a severe, rare complication of deep vein thrombosis, which is characterised by compartment syndrome, arterial compromise, venous gangrene, and shock. Prothrombotic states are the primary risk factor for PCD, which, in most cases, is associated with pulmonary embolism and carries a high mortality.
\end{abstract}

Case report: A 46-year-old male presented following a pulseless electrical activity (PEA) arrest due to saddle pulmonary embolism (PE). He subsequently developed PCD and venous gangrene secondary to inferior vena cava obstruction, in the setting of a new diagnosis of testicular germ cell tumour.

Discussion: PEA arrest, as the initial presenting problem in malignancy, is rare. It is extreme for the first indication of cancer to be a PEA arrest from massive PE. While hypoxic brain injury from the cardiac arrest precluded intervention in this case, a surgical approach entailing en bloc resection of aortocaval metastasis, with subsequent IVC reconstruction, followed by lower limb venous thrombectomy would have been favoured as it was considered that an endovascular approach would not have been successful.

Conclusion: A case of a patient with phlegmasia cerulea dolens secondary to testicular cancer, who presented following PEA arrest is described.

Keywords: phlegmasia cerulea dolens, pulseless electrical activity arrest, pulmonary embolism, testicular cancer

Received: 10 April 2019 / Accepted: 25 April 2019

\section{INTRODUCTION}

Phlegmasia cerulea dolens (PCD) is a severe, rare complication of deep vein thrombosis (DVT), which is characterised by compartment syndrome, arterial compromise, venous gangrene, and shock [1]. PCD is caused by extensive DVT which extends into collateral veins, causing total venous obstruction [2]. Subsequent venous congestion causes elevated intravascular pressure leading to extravasation of plasma and significant oedema. Compartment syndrome follows, resulting in arteriole collapse and ischaemia, and subsequent venous gangrene $[1,2]$. Though first described in the $16^{\text {th }}$ century, it was in 1938 that Grégoire defined phlegmasia cerulea dolens as a clinical triad of cyanosis, acute limb swelling and ischaemic pain [1]. The risk factors for PCD include prothrombotic states such as malignancy, pregnancy, heparin-induced thrombocytopenia and antiphospholipid syndrome, as well as other fac- tors such as external venous compression and femoral vein catheterisation $[3,4]$. The condition is associated with pulmonary embolism (PE) in most cases and has a high mortality $[3,5]$.

Duplex ultrasonography is usually the first imaging modality in the diagnosis of PCD, due to its accessibility, reliability, and non-invasive nature. CT and MR venography are useful adjuvant modalities to determine the extent of thrombus and potential underlying causes, including extrinsic venous compression [1]. The gold standard imaging modality is considered to be CT venography [6].

The management of PCD involves a combination of medical and surgical therapies depending on the effectiveness of treatment. The goal of any initial treatment is to halt the progression of thrombus formation to preserve the patency of any remaining collateral veins, and consists of systemic anticoagulation [7]. If this does not 
result in clinical improvement, further intervention is then required to remove the venous obstruction $[1,2]$. This may consist of catheter-directed thrombolysis or surgical venous thrombectomy. Surgical intervention may be favoured in situations where thrombolysis is contraindicated, or when the clinical situation is such that immediate action is required for limb salvage. If these measures are unsuccessful venous bypass with an arteriovenous fistula can be attempted [1]. Adjunctive measures include placement of an inferior vena cava (IVC) filter, and fasciotomy [8]. Given the high incidence of PE with PCD, IVC filter placement is an important measure to prevent further $\mathrm{PE}$ that may be a complication of treatment.

\section{CASE REPORT}

A previously healthy 46-year-old male, on no medications, presented following a pulseless electrical activity (PEA) arrest after collapsing at home. He was resuscitated and had the return of spontaneous circulation before arriving at the nearby tertiary care hospital. The patient underwent a CT pulmonary angiogram and $\mathrm{CT}$ of the abdomen and pelvis which demonstrated large saddle PE (Figure 1) with a significant burden of thrombus extending into both right and left pulmonary artery circulations. Evidence of right heart strain was demonstrated on a bedside echocardiogram. The patient was treated with therapeutic anticoagulation with an intravenous heparin sodium (Pfizer, Sydney, Australia) infusion, insertion of an IVC filter and pulmonary rheolytic thrombectomy. He was subsequently managed in intensive care while intubated, requiring minimal inotropic support with intravenous norepinephrine (Pfizer, Sydney, Australia) infusion.

A testicular mass on CT and elevated levels of alphafetoprotein revealed a diagnosis of germ cell tumour, which had not been previously diagnosed. CT also demonstrated a significant burden of thrombus within the IVC and iliac veins (Figure 2), with an associated aortocaval lesion in direct continuity with the IVC.

The patient subsequently developed venous gangrene, with bilateral cold, mottled lower limbs, and tight compartments with no dorsalis pedis or posterior tibial artery pulses. Bilateral lower limb duplex ultrasound demonstrated the presence of extensive bilateral lower limb venous thrombosis extending to the IVC with no evidence of arterial thrombosis. He was consequently diagnosed with phlegmasia cerulea dolens sec- ondary to inferior vena cava obstruction, in the setting of testicular germ cell tumour.

The treatment options considered included open or endovascular reconstruction of the inferior vena cava. Unfortunately, the patient did not display significant neurological recovery following the cardiac arrest, with

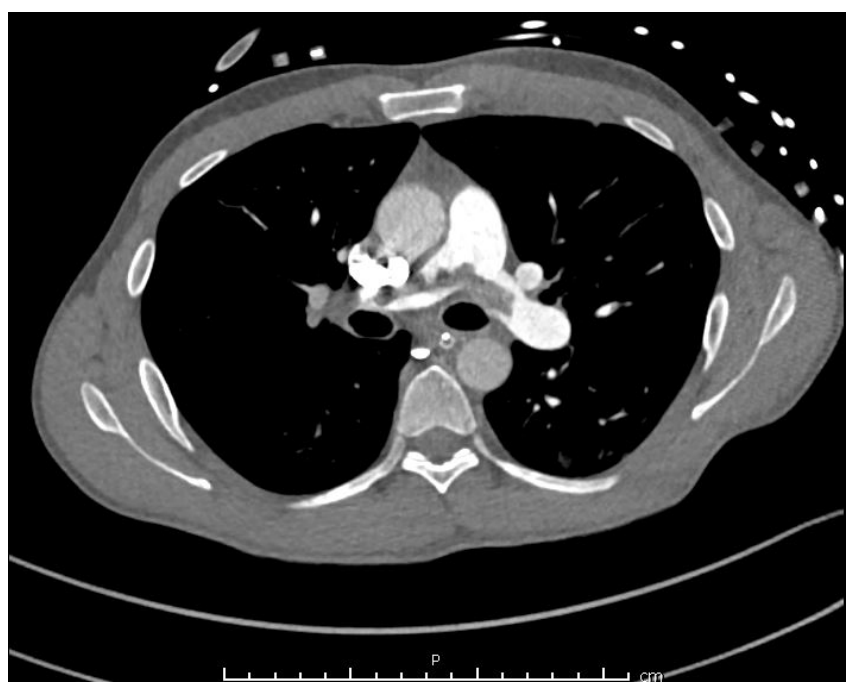

Fig. 1. CT pulmonary angiogram axial image demonstrating saddle pulmonary embolism

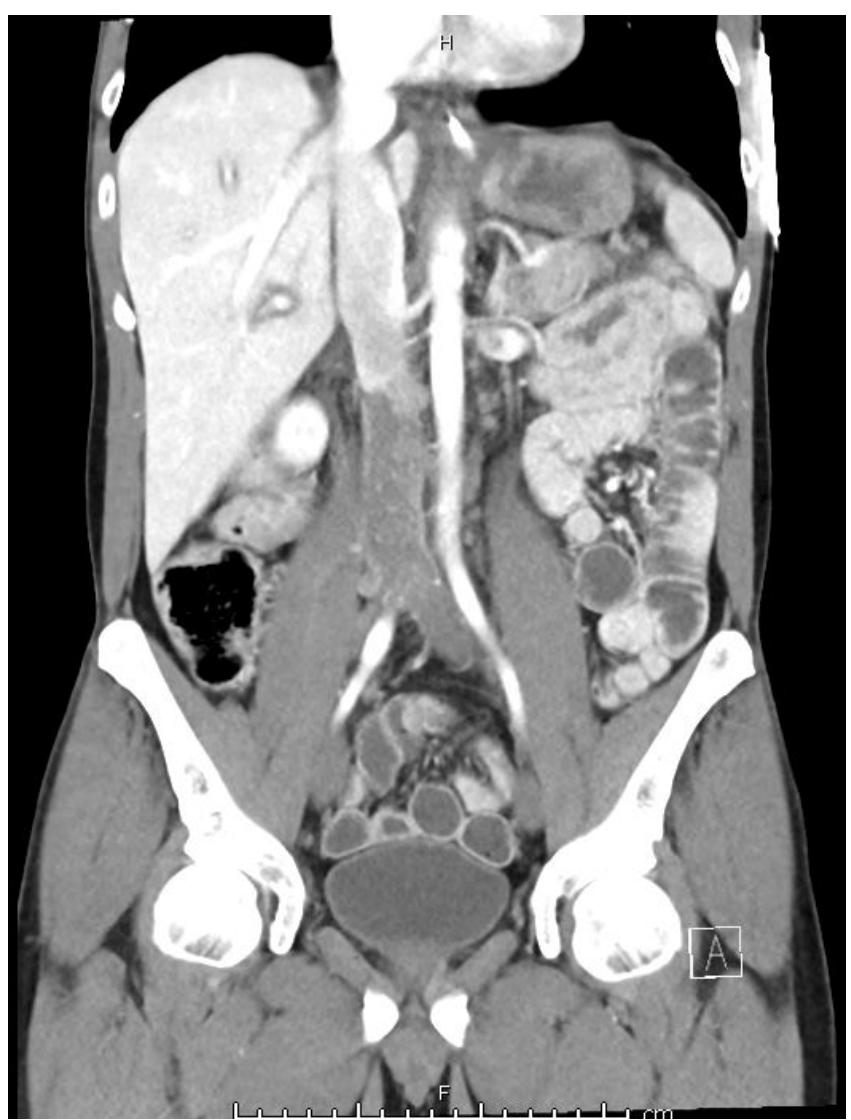

Fig. 2. CT abdomen and pelvis coronal image demonstrating extensive iliocaval thrombosis 
electroencephalogram and CT brain findings consistent with catastrophic hypoxic brain injury, with no likelihood of meaningful neurological recovery. The patient was subsequently palliated, with withdrawal of active treatment including inotropic support, renal replacement therapy, enteral feeding, and died shortly thereafter in the intensive care unit.

\section{DISCUSSION}

Venous thromboembolism (VTE), including DVT and $\mathrm{PE}$, is a common complication of malignancy $[9,10]$. PEA arrest is a recognised complication of a massive PE $[10,11]$. However, PEA arrest, as the initial presenting problem in malignancy, is rare. It is uncommon for the first indication of cancer to be a PEA arrest from a massive PE.

The hypoxic brain injury suffered by this patient deemed any intervention in the management of his PCD to be futile to the overall outcome. However, before a prognosis was established, active management options were considered, mainly since germ cell tumours are associated with a good prognosis if patients receive active treatment [12]. Surgical and endovascular options were considered. While surgical resection is not required for all germ cell tumour metastases following chemotherapy [13], in this instance, due to the retroperitoneal aortocaval lesion with invasion into the IVC, a surgical approach would have required en bloc resection of the aortocaval metastasis with subsequent IVC reconstruction, followed by lower limb venous thrombectomy to clear the lower limb venous obstruction. An endovascular approach would have involved catheter-directed thrombectomy and thrombolysis with the likelihood of stenting to re-canalise the IVC and lower limb deep venous system. Surgical intervention would have been favoured as it was considered that an endovascular approach would not have been successful given the significant thrombus burden.

The combination of the prothrombotic state of malignancy, coupled with the compression and invasion of tumour metastasis into the IVC likely caused the significant degree of thrombus in this patient and the subsequent IVC obstruction. It was this IVC obstruction that lead to bilateral PCD and venous gangrene.

This case is unusual in that PCD most often presents unilaterally, due to iliac or femoral vein thrombosis $[1,14]$. Iliac vein DVT is commonly caused by compression, particularly with pelvic masses such as large uterine fibroids. However, the progression to PCD in these cases is exceedingly rare [8].

The presentation of VTE, such as DVT and PE, warrants careful search for a precipitating cause, including malignancy, particularly with an atypical or aggressive clinical presentation of thrombus [1]. VTE may be the first presentation of malignancy and may precede the diagnosis of cancer by months or even years [1].

\section{-CONCLUSION}

Phlegmasia cerulea dolens is a severe complication of deep vein thrombosis and carries a high mortality, especially once venous gangrene develops. It is a rare condition often associated with prothrombotic states such as malignancy. Management is challenging, graded and has to be enacted early. This is a rare case of a patient with phlegmasia cerulea dolens secondary to testicular cancer, who presented following PEA arrest.

\section{口CONCLICT OF INTEREST}

None to declare.

\section{REFERENCES}

1. Chang G, Yeh JJ. Fulminant phlegmasia cerulea dolens with concurrent cholangiocarcinoma and a lupus anticoagulant: a case report and review of the literature. Blood Coagul Fibrinolysis. 2014;25(5):507-11.

2. Vysetti S, Shinde S, Chaudhry S, Subramoney K. Phlegmasia cerulea dolens - a rare, life-threatening condition. Scientific World Journal. 2009;9:1105-6.

3. Garcia-Fernandez-Bravo I, Ordieres-Ortega L, Toro-Cervera D. Cancer associated phlegmasia cerulea dolens successfully treated with apixaban. Blood research. 2018;53(1):90-2.

4. Mumoli N, Invernizzi C, Luschi R, Carmignani G, Camaiti A, Cei M. Phlegmasia cerulea dolens. Circulation. 2012;125(8):10567.

5. Maiti A, Das A, Smith DT. Phlegmasia cerulea dolens. Postgrad Med J. 2016;92:690.

6. Chinsakchai K, ten Duis K, Moll FL, de Borst GJ. Trends in management of phlegmasia cerulea dolens. Vasc Endovasc Surg. 2011;45(1):5-14.

7. Weaver FA, Meacham PW, Adkins RB, Dean RH. Phlegmasia cerulea dolens: therapeutic considerations. South Med J. 1988;81(3):306-12.

8. Brewer MB, Woo K, Weaver FA. Venous thromboembolism secondary to uterine fibroids: a case of phlegmasia cerulea dolens and review of the literature. Ann Vasc Surg. 2015;29(2):364 e5-e9. 
Available online at: www.jccm.ro

9. Prandoni P, Piccioli A, Girolami A. Cancer and venous thromboembolism:

an overview. Haematologica. 1999;84(5):437-45

10. Carson JL, Kelley MA, Duff A, Weg JG, Fulkerson WJ, Palevsky HI, et al. The clinical course of pulmonary embolism. N Engl J Med. 1992;326(19):1240-5.

11. Hess EP, Campbell RL, White RD. Epidemiology, trends, and outcome of out-of-hospital cardiac arrest of non-cardiac origin. Resuscitation. 2007;72(2):200-6.
The Journal of Critical Care Medicine 2019;5(2) • 59

12. Horwich A, Shipley J, Huddart R. Testicular germ-cell cancer. The Lancet. 2006;367(9512):754-65.

13. Hussain SA, Ting Ma Y, Cullen MH. Management of metastatic germ cell tumors. Expert review of anticancer therapy. 2008;8(5):771-84.

14. Kamphol Laohapensang M, Hanpipat S, Aworn S, Orrapin S. Surgical venous thrombectomy for phlegmasia cerulea dolens and venous gangrene of the lower extremities. J Med Assoc Thai. 2013;96(11):1463-9. 